\section{Audiovisuelle Fragebögen: Der MultiMedia-Computer als Erhebungsinstrument in der Musik- und Medienforschung}

\section{RENATE MÜLLER}

Ich stelle hier ein Forschungsinstrument im Kontext meiner eigenen Forschungen vor, daher gehe ich

1. auf den Forschungskontext ein.;

2. werde ich eingehen auf die Bedeutung audiovisueller Fragebögen und präsentativer Methoden der Sozialforschung.

3. werde ich anhand einiger Beispiele aus meinem VideoCultureFragebogen das Fragebogen-Autorensystem FrAuMuMe darstellen;

4. Gehe ich ein auf damit verbundene neue Formen der Präsentation von Ergebnissen: Präsentative Methoden und die Präsentation von Ergebnissen.

\section{Folie 1:}

\section{Audiovisuelle Fragebögen: \\ Der MultiMedia-Computer als Erhebungs- instrument in der Musik- und Medienfor- schung}

1. Der Forschungskontext: VideoCulture

2. Zur Bedeutung audiovisueller Fragebögen und präsentativer Methoden in der Sozialforschung

3. Das Fragebogen-Autorensystem FrAuMuMe an Beispielen aus dem Videofragebogen

4. Ergebnispräsentationsmöglichkeiten

\section{Der Forschungsk ontext}

Es wird ein Forschungsinstrument vorgestellt, eng verknüpft mit einem aktuellen Forschungsprojekt. Dennoch geht es hier um das Forschungsinstrument und weniger um die Darstellung inhaltlicher Fragen und der ersten Forschungsergebnisse aus dem Projekt V ideoC ulture auf dem MultiM edia-Computer: A udiovisuelle Kommunik ation Jugendlicher.

Im Projekt V ideoC ulture produzieren Jugendliche in verschiedenen Ländern (D eutschland, England, Ungarn, Tschechei und USA) Videos ohne Sprachanteile, die auf der Grundlage der Theorie einer interkulturell verständlichen audiovisuellen Symbolsprache Jugendlicher analysiert werden. D as Teilprojekt V ideoC ulture auf dem M ultiM edia-Computer verfolgt $u$. a. die folgenden Zielsetzungen mit dem MultiMediaComputer als Erhebungsinstrument.
1. Die Anziehungskraft audiovisueller Symbole wird im Hinblick darauf untersucht, ob die Befragten in ihrer Attraktivitätszuschreibung an audiovisuelle Symbole übereinstimmen.

2. Verschiedene Typen der Videorezeption mit jeweils unterschiedlichen inhaltlichen Präferenzen werden herausgearbeitet.

3. Das Forschungsinstrumentarium wird im Hinblick darauf exploriert, ob es geeignet ist, Übereinstimmungen und Unterschiede in der Beurteilung und im Erleben audiovisueller Symbole von Jugendlichen in den Blick zu nehmen.

Die Fragestellungen dieser Studie ergeben sich u. a. aus den Konzepten zur musikalischen Selbstsozialisation und selbstsozialisierten audiovisuellen Kompetenz. Diese sind eng verknüpft mit der Vorstellung, dass mit der kulturellen Differenzierung durch Medien die Entwicklung neuer Formen der Weltaneignung einhergeht, die weniger durch Regeln sprachlicher Diskursivität als durch Analogik, präsentative Symbolik bestimmt sind. D araus ergibt sich die Anforderung an die Erforschung des Umgehens mit Musik und Medien, sich auf vorhandene präsentative Forschungsmethoden zu besinnen und weitere Formen präsentativer Forschung zu entwickeln.

Präsentative Methoden der Sozialforschung: Audiovisuelle Fragebögen

Methoden der empirischen Sozialforschung: Befragung ist die am häufigsten eingesetzte Methode.

Folie 2:

Methoden der empirischen Sozialforschung

1 Die Befragung

Mündliche Befragung: das Interview

Persönliche Befragung

Computer Assisted Interview CAI

Telefonbefragung CATI

Schriftliche Befragung: der Fragebogen

Papier und Bleistift-Befragung PAPI

Postalische Befragung

Computer Assisted Selfadministered

Interview CASI

Internet-Befragung

2 Das Experiment

3 Die Beobachtung

„Unter Interview als Forschungsinstrument sei hier verstanden ein planmäßiges Vorgehen mit wissenschaftlicher Zielsetzung, bei dem die Versuchsperson durch eine Reihe gezielter Fragen oder mitgeteilter Stimuli zu verbalen Informationen veranlasst werden soll."

Erwin K. Scheuch 1973 
Für Untersuchungen zur audiovisuellen Sozialisation und zur Wirkung audiovisueller Symbole sind Forschungsmethoden geeignet, die möglichst nah an die ästhetischen Entscheidungen und das audiovisuelle Material herankommen. Mit präsentativen Forschungsmethoden werden Musikstücke, Bilder und Videoclips vorgeführt, um Musik-, Kunst- und Filmerleben zu untersuchen. Präsentative Methoden beschränken sich nicht auf verbale Impulse zur Untersuchung audiovisueller Präferenzen. Darüber hinaus engen sie die Befragten nicht darauf ein, verbale Informationen geben zu müssen.

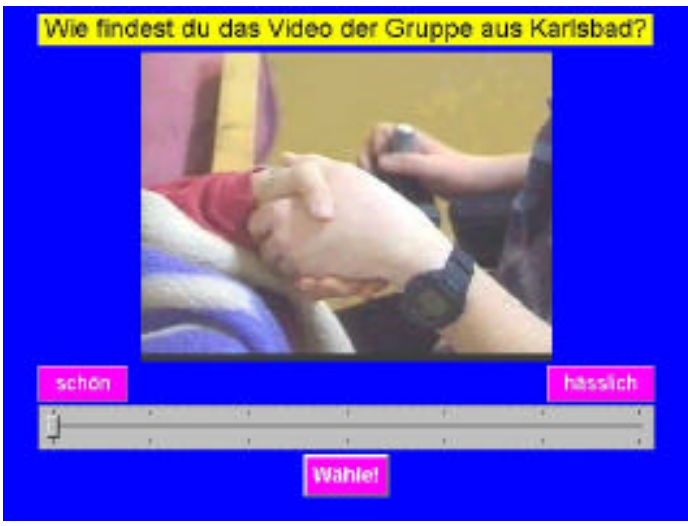

In Verbindung mit der Präsentation von Musik, Bild und Film eignen sich besonders Semantische D ifferentiale dazu, audiovisuelles Erleben unmittelbar am audiovisuellen Objekt und weitgehend unabhängig vom Verbalisierungsvermögen der Befragten zu untersuchen. Mit semantischen Differentialen (Polaritätsprofilen, Eindrucksprofilen) können Befragte auf einer Skala zwischen Gegensatzpaaren (Polaritäten) ihre Eindrücke von einem ästhetischen Objekt, z. B. einem Bild, einem Musikstück, einem Video, einordnen - ohne ihre Wahrnehmungen, Gefühle, Assoziationen selbst zu verbalisieren. Die Methode wird $u$. a. in der Kunst- und Musikrezeptionsforschung und in der Imageforschung angewendet, um Anmutungen der Befragten Objekten gegenüber zu erheben und zu vergleichen.

Im vorliegenden VideoCulture-Fragebogen werden nach der jeweiligen Präsentation eines der fünf Videos zu Beginn des Fragebogens Anmutungen der Videos und ihre erlebte Attraktivität anhand des Semantischen Differentials aus acht Eigenschaftspaaren über jeweils siebenstufige Skalen erhoben:

verständlich - unverständlich, interessant - langweilig,

wichtig - unwichtig,

vertraut - ungewohnt,

schön - hässlich,

fröhlich - traurig,

toll - schlecht,

anziehend - abstoßend.

Die Videopräsentation erfolgt in zufälliger Reihenfolge, um den MedienReiheneffekt zu kon- trollieren. Jedem Befragten werden die acht Eigenschaftspaare ebenfalls in zufälliger Reihenfolge präsentiert, um den FrageReiheneffekt zu kontrollieren; die Reihenfolge wird aber für jedes Individuum über alle fünf Videos hinweg beibehalten.

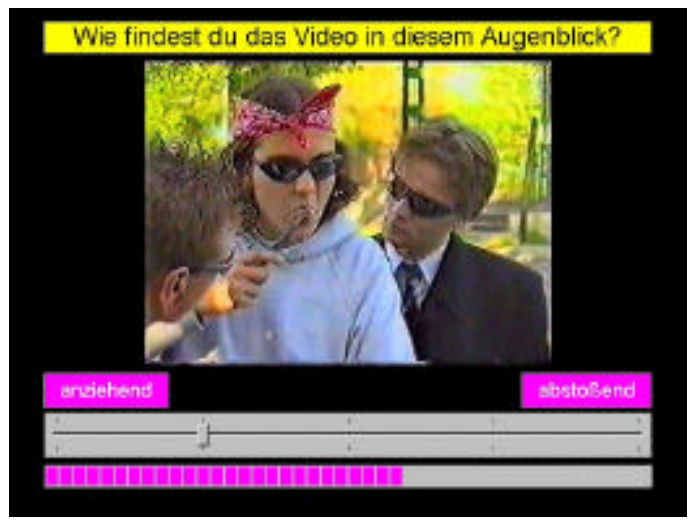

Auch durch die Untersuchung kontinuierlicher Antworteingaben (Continuous Response Measure ment, CRM) während der Musik- oder Videorezeption werden Möglichkeiten geschaffen, audiovisuelles Erleben zu quantifizieren.

Wenn es den Befragten möglich gemacht wird, ihre Eindrücke von einem Musikvideo oder einem Werbespot ablaufsimultan während der Präsentation des Clips kontinuierlich rückzumelden, sprechen wir von der Methode des Continuous Response, die insbesondere in der Kommunikations- und in der Musikforschung eine Rolle spielt.

Dabei können die Reaktionen der Befragten auf den Clip oder Spot unter verschiedenen Gesichtspunkten eingegeben werden, sei es unter dem Aspekt ihres Gefallens an dem Video, der durch das Video erzeugten Spannung, der dramaturgischen Bedeutsamkeit des jeweiligen Augenblicks für das gesamte Video oder, wie in der vorliegenden Untersuchung, unter dem $\mathrm{Ge}$ sichtspunkt des Fasziniert- oder Abgestoßenseins durch ein Video. Die Befragten konnten sich das Video selbst zum nochmaligen Ansehen aussuchen. Im aktuellen Fragebogen entscheiden sie sich kontinuierlich auf einer fünfstufigen Skala zwischen den Gegensätzen „anziehend - abstoßend“. Die kontinuierliche Aufzeichnung der Antworten ermöglicht es, über punktuelle Gesamtäußerungen hinaus Veränderungen der Beurteilung des Videos über seinen Verlauf hinweg in den Blick zu nehmen.

VideoCulture auf dem MultiMedia-Computer und das Fragebogen-A utorensystem FrA uMuMe

Der MultiMedia-Computer als Erhebungsinstrument präsentiert audiovisuelle Fragebögen und initiiert und untersucht ästhetische Entscheidungsprozesse der Befragten. 


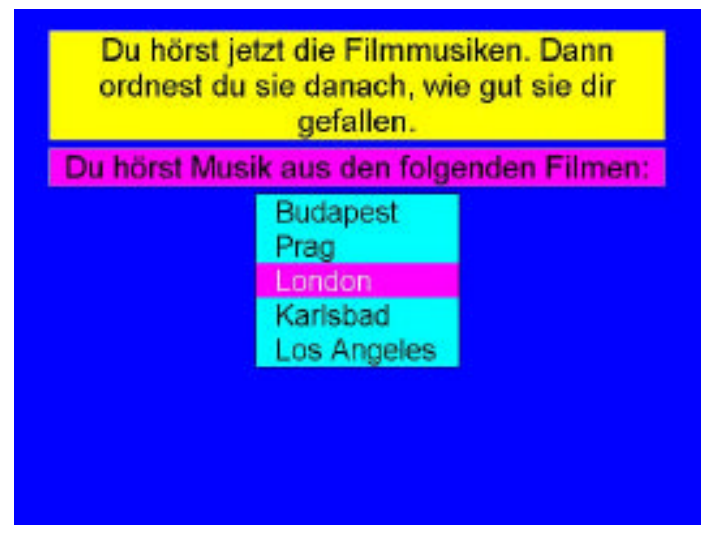

Ein audiovisueller Fragebogen wurde entwickelt, mit dem Jugendliche am Computer u. a. darüber befragt werden, wie sie die Videos anderer Jugendlicher entlang verschiedener Erlebnisdimensionen beurteilen. D er Fragebogen ist so aufgebaut, dass Videos und Musikstücke nach verschiedenen Dimensionen beurteilt und erlebt werden können. Erhoben werden ästhetische und Präferenzurteile, Wahrnehmungsurteile, reale Entscheidungen für Videos, Bilder und Musikstücke, Anmutungen und Bedeutungszuschreibungen - jeweils eng verknüpft mit der unmittelbaren Präsentation von Musik, Bildern und Videos. Hier werden z.B. "klassische" Präferenzurteile anhand eines MusikRanking erhoben, wobei jeweils über fünf Objekte entschieden wird, welches am besten und am zweitbesten gefällt und so fort.

Bei dem Fragebogen-Autorensystem MultiMedia FrA uMuMe handelt es sich um ein umfassendes Befragungssystem:

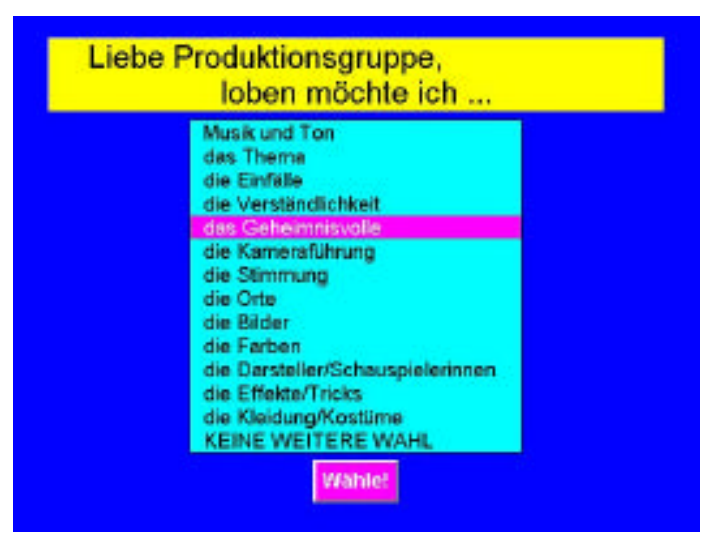

1. Es enthält bewährte verbale und präsentative Fragetypen, z. B. MultipleChoiceFragen.

2. Klingende, visuelle und audiovisuelle Fragefeatures werden integriert.

3. Mit FrAuMuMe kann eine nahezu unbegrenzte Anzahl verschiedener Fragebögen auf dem MultiMedia-Computer erzeugt werden. Insbesondere Studierende benut- zen es für ihre Examens- und DiplomArbeiten, sei es zum Thema Identitätskonstruktion durch die Rezeption der Dr. Sommer-Seite in der BRAVO oder durch parasoziale Interaktion mit den D ailySoaps oder zur Frage der kulturellen Aktivitäten von Teenie-Fans. (vgl. „Studentische Forschungsvorhaben mit dem Forschungsinstrument FrA uMuM e')

Es handelt sich hier um ein vollständiges Befragungssystem, das den Computereinsatz nicht auf die Präsentation von audiovisuellem Material beschränkt, wie es in der Musikforschung üblich ist. Dadurch wird es möglich, so verschiedene Daten zu erfassen und zueinander in Beziehung zu setzen wie Weisen audiovisuellen Erlebens auf der einen Seite und demographische Daten, D aten zur Medienerfahrung, zum Freizeitverhalten, zur jugendmusikkulturellen Orientierung und zu fankulturellen Aktivitäten und Einstellungen auf der anderen Seite.

Erst durch Multimedia wird die Integration von Text, Sound, Bild, Video und interaktivem Vorgehen möglich:

- Text beinhaltet z. B. Fragen und Informationen bzw. Instruktionen.

- Sound meint vor allem „richtige" Musikbeispiele, die während der Befragung über Kopfhörer gehört werden. In der vorliegenden Untersuchung wurden die „Soundtracks” der jugendlichen Videoproduktionen auch als zu beurteilende Musikstücke verwendet, sei es zur Erhebung von MusikPräferenzen wie hier oder als

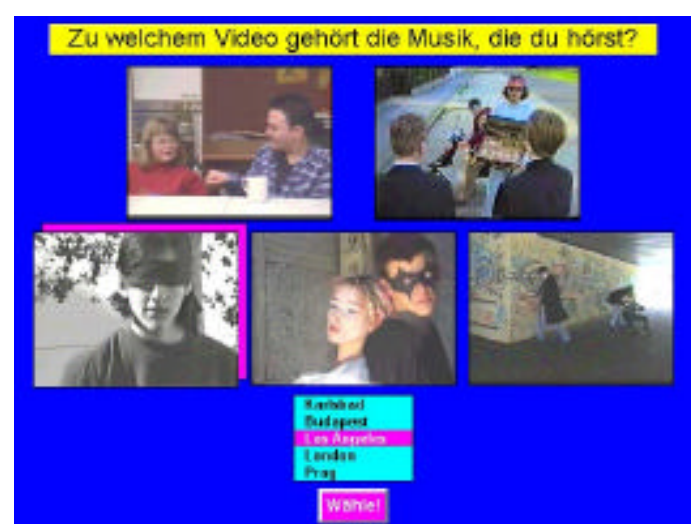

MusikQuiz wie hier, wo die Befragten die Soundtracks den Videos zuordnen sollen und Musik und Bild wiedererkennend verknüpfen.

- Bild beinhaltet Grafik bis hin zu Fotos und Animation, im VideoCulture Fragebogen insbesondere die aus den Videos gegrabbten Bilder.

- Video bezieht sich auf Ton bzw. Musik und Film (Videoclips und Werbespots). In den VideoCulture-Fragebogen wurden fünf jugendliche Videoproduktionen, aus Budapest, Karlsbad, London, Los Angeles und Prag, einbezogen. 


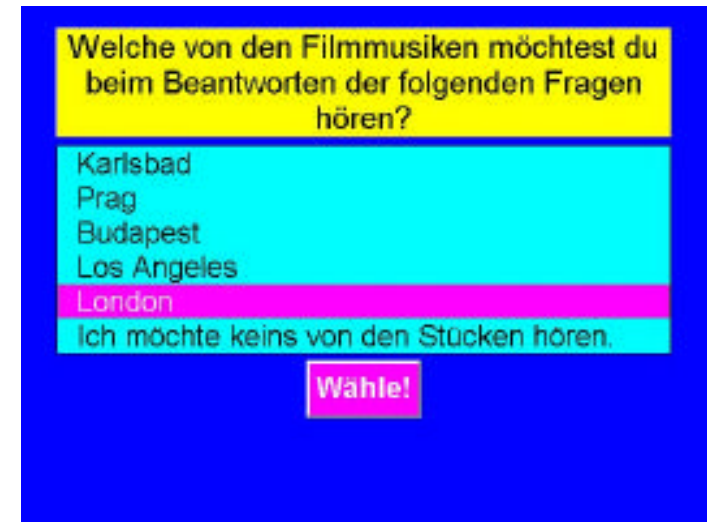

Interaktiv reagiert der Computer $\mathrm{z}$. B. dort auf die Befragten, wo sie entscheiden können, ob während der Beantwortung von Wortfragen zum Freizeitverhalten die Filmmusiken aus den Videos gespielt werden sollen oder nicht.

Als "offenbarte Präferenz" wird hier die Zuneigung zu den Filmmusiken erhoben, indem die Option gegeben wird, sich zweimal einen der Soundtracks noch einmal anzuhören (Mood Managing).

Interaktiv reagiert der Computer auch auf Antworten von Filterfragen und Bereichsfragen, wo je nach Antwort zu verschiedenen Fragen gesprungen wird. Z. B. können sich die Befragten im vorliegenden Fragebogen für ein Video entscheiden, das sie noch einmal sehen können und zu dem ihnen Fragen gestellt werden.

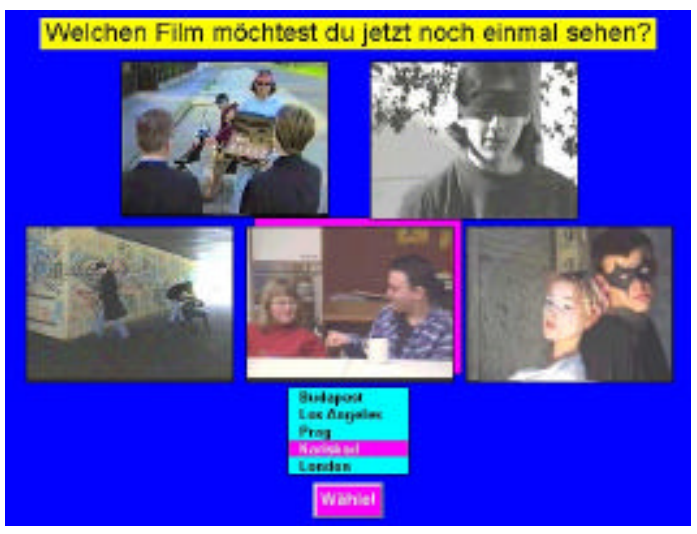

Im Fragebogen-Autorensystem FrAuMuMe werden die vollständige Präsentation der Fragen, der musikalischen Ereignisse, der Bilder und Videos sowie die Datenerfassung und die Vorbereitung der Datenauswertung vom Computer gesteuert und ausgeführt, von

- der Randomisierung, der zufälligen Anordnung von Fragen, Musikstücken, Videos und Bildern,

- der Messung von Antwortzeiten,

- der Instruktion incl. der Aufgabe zum Üben für die Befragten, die selbständig und allein ihre Antworten in den Computer eingeben,
- der Präsentation von Informationen, Fragen, experimentellen Designs, audiovisuellem Material,

- der Antworteingabe,

- der Datenaufzeichnung bis hin

- zur Vorbereitung der Datenauswertung durch Erstellung einer SPSS-D atalist incl. Variable-Lables und Value-Lables.

Wenn wir eine Woche lang mit vier Computern in einer Schule waren, haben wir Freitag Abend 130 Datensätze mit 700 Variablen als SPSSDatensatz.

Der MultiMedia-Fragebogen erleichtert es, präsentative Befragungsmethoden in der empirischen Sozialforschung einzusetzen, weil die Handhabung des visuellen, musikalischen und audiovisuellen Materials sehr vereinfacht wird. Darüber hinaus wird der sprachliche Anteil des Fragebogens durch die großen visuellen und auditiven Anteile gering gehalten und es werden geringe Anforderungen an das Sprachvermögen der Befragten bei ihrer Antworteingabe gestellt. So versucht das Forschungsinstrument der eingangs skizzierten Verschiebung von Wahrnehmungs- und Deutungssystemen weg von der diskursiven hin zur präsentativen Logik gerecht zu werden. Die Befragten können ihre Eindrücke von audiovisuellen Medien wiedergeben, ohne dass diese verbal repräsentiert werden, sondern indem vor allem audiovisuell auf sie verwiesen wird.

\section{Präsentative Methoden und Präsentation ihrer Ergebnisse}

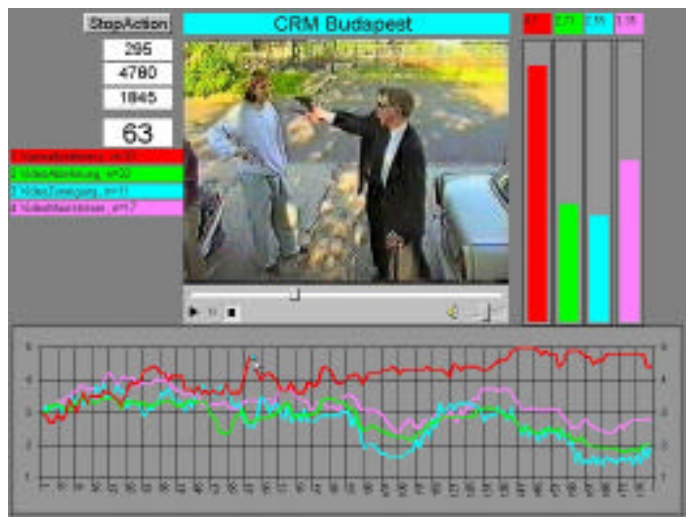

Zur Auswertung und D arstellung der Ergebnisse des CRM wird das Präsentationsprogramm JustForPlay verwendet.

\section{Zum Schluss}

Eine erste Teilstudie mit 134 befragten Achtklässlem ergab, dass der benutzte audiovisuelle Fragebogen insbesondere durch die Verwendung von CRM neue Einblicke in die Videobeurteilung durch Gruppierungen Jugendlicher ermöglicht. Dabei konnte zum einen gezeigt werden, dass es übereinstimmende jugendliche Reaktionen auf die in den jugendlichen Video- 
produktionen verwendeten audiovisuellen Symbole gibt. Dies kann gedeutet werden als eine Bestätigung der Vermutung, dass Jugendliche sich einer interkulturell verständlichen audiovisuellen Symbolsprache bedienen. Zum anderen konnten durch Clusteranalysen Typen der Videorezeption voneinander unterschieden werden, die audiovisuelle Symbole verschieden beurteilen und die sich u.a. im Hinblick darauf voneinander differenzieren lassen, wie stark sie sich von Video-Gewalt und subkultureller Symbolik angezogen oder abgestoßen fühlen.

\section{Studentische Forschungsvorhaben mit dem Forschungsinstrument FrAuMuMe}

Kreutle, Andreas, 1997: Femsehen und Identität. Eine empirische Untersuchung zur Mediennutzung Jugendlicher und ihre pädagogischen Konsequenzen. Wissenschaftliche Hausarbeit zur Ersten Staatsprüfung für das Lehramt an G rund- und Hauptschulen. Pädagogische Hochschule Ludwigsburg.

Glogner, Patrick, 1998: Der Beitrag der Dr.Sommer-Beratung zur Identitätsbildung Jugendlicher. Eine empirische Untersuchung zur Rezeption der Jugendzeitschrift BRAVO und ihre pädagogischen Konsequenzen. Wissenschaftliche Hausarbeit zur Ersten Staatsprüfung für das Lehramt an Grund- und Hauptschulen. Pädagogische Hochschule Ludwigsburg.

Eber, Erich, 1998: Zum Einfluß der LehrerSchüler-Beziehung auf die Rezeptionsbarrieren Jugendlicher. Eine empirische Untersuchung zur musikalischen Sozialisation im Musikunterricht. Wissenschaftliche Hausarbeit zur Ersten Staatsprüfung für das Lehramt an Realschulen. Pädagogische Hochschule Ludwigsburg.

Ebert, Sabine, 1998: Femsehserien als Anlaß zur Selbstreflexion? Eine empirische Untersuchung zur Rezeption von Fernsehserien durch Jugendliche und ihre pädagogischen Konsequenzen. Wissenschaftliche Hausarbeit zur Ersten Staatsprüfung für das Lehramt an Grundund Hauptschulen. Pädagogische Hochschule Ludwigsburg.

D ongus, Nadine, 1999: Selbstsozialisation durch den Computer? Eine empirische Untersuchung zur Computernutzung von Kindern und Jugendlichen und ihre pädagogischen Konsequenzen. Wissenschaftliche Hausarbeit zur Ersten Staatsprüfung für das Lehramt an G rund- und Hauptschulen. Pädagogische Hochschule Ludwigsburg.

Annette Würth, 1999: Wie erfahren Lehreninnen und Lehrer von Forschungsergebnissen? Diplom-Arbeit/ Schulpädagogik. Pädagogische Hochschule Ludwigsburg.

Stefanie Rhein, 1999: Jugendliche Musikfans und die fanspezifische N utzung populärmusikalischer Angebote. Eine empirische Untersuchung mit dem MultiMediaComputer. Magisterarbeit Kulturmanagement. Pädagogische Hochschule Ludwigsburg.

Heim, Jens, 2000: Zur audiovisuellen Kompetenz von Viertklässlem, Achtklässlem und Studierenden. Eine empirische Untersuchung mit dem MultiMedia-Computer. Wissenschaftliche Hausarbeit zur Ersten Staatsprüfung für das Lehramt an Grund- und Hauptschulen. Pädagogische Hochschule Ludwigsburg. 
Weißenburger, Christian, 2001: Zur Wirkung von Musik in der Werbung. Eine experimentelle Untersuchung mit dem MultiMediaComputer zum Verhältnis von Auge und $\mathrm{Ohr}$ bei SchülerInnen der Jahrgangsstufen 8-10. Wissenschaftliche Hausarbeit zur Ersten Staatsprüfung für das Lehramt an Realschulen. Pädagogische Hochschule Ludwigsburg.

Heim, Jens, 2002: Audiovisuelle Kompetenz in der Grundschule. Eine empirische Untersuchung über eine computerunterstützte medienpädagogische Unterrichtseinheit zum Umgang von Viertklässlern mit bewegten Bildern und Musik. Wissenschaftliche Hausarbeit zur Magisterprüfung für den Magisterstudiengang mit Schwerpunkt Fachdidaktik. Pädagogische Hochschule Ludwigsburg.

\section{Veröffentlichungen studentischer Forschungsvorhaben mit dem For- schungsinstrument FrA uMuMe}

Glogner, Patrick: Selbstsozialisation und Identitätskonstruktion mit der D r.-Sommer-Seite. Eine Befragung mit dem MultiMedia-Computer zur Rezeption der Jugendzeitschrift BRAVO. In: deutsche jugend. Heft 7/ 8, 2000, 318-326.

Kreutle, Andreas: Jugendliche Identitätsbildung durch Fernsehen. In: tv diskurs. Heft 7, 1999, 42-52.

Müller, Renate \& Nadine D ongus, Sabine Ebert, Patrick G logner, Andreas K reutle: Identitätskonstruktion mit Medien und Musik. Ein empirisches Forschungsprogramm mit MultiMediaFragebögen. In: medien praktisch 1999, 22. Jg., Heft 1, 26-30.

Müller, Renate, Patrick Glogner, Jens Heim, Stefanie Rhein \& Christian Weißenburger. "Studierende forschen mit dem MultiMediaComputer. Ein Lehr- und Forschungskonzept." Januar 2001. http:/ / www.ph-karlsruhe.de/ HDT/ hdt-berichte.htm.

Rhein, Stefanie: Teenie-Fans: Stiefkinder der Popularmusikforschung. Eine Befragung Jugendlicher mit dem MultiMediaComputer über ihre Nutzung fankultureller Angebote. In: Werner Heinrichs \& Armin Klein (Hrsg.): D eutsches Jahrbuch für Kulturmanagement 1999, Band 3, Baden-Baden 2000, S. 165-194. 